\title{
Rehabilitative management of pelvic fractures needs to be further optimized
}

\author{
Zehua Li (1), Dilihumaer Maimaitiming (2), Leqi Sun (3), Haoran Wang (1), Weixi \\ Xiong $(4,5)$
}

(1) Department of Burn and Plastic Surgery, West China Hospital of Sichuan University, Chengdu, China; (2) West China School of Medicine, Sichuan University, Chengdu, China; (3) Queen Square Institute of Neurology, University College London (UCL), London, UK; (4) Department of Neurology, West China Hospital of Sichuan University, Chengdu, China; (5)Institute of Brain Science and Brain-inspired technology of West China Hospital, Sichuan University, Chengdu, China.

This article is distributed under the terms of the Creative Commons Attribution Noncommercial License (CC BY-NC 4.0) which permits any noncommercial use, distribution, and reproduction in any medium, provided the original author(s) and source are credited.

\begin{abstract}
We read with interest the review by Piccione et al. into the rehabilitative management of patients with pelvic fracture (PF). This review adds to our knowledge about the significance and indispensability of early multidisciplinary intervention in PF. From our perspective, however, potential bias might be caused by several unanswered questions. The uncertain methodological process and the unclear definition could misguide the rehabilitation strategies while still in dispute. Therefore, further high-quality studies should be conducted to optimize the multidisciplinary rehabilitation of patients with PF.
\end{abstract}

Key Words: Pelvic; acetabular; fractures; rehabilitation.

Eur J Transl Myol 31 (4): 10246, 2021 doi: 10.4081/ejtm.2021.10246

$\mathbf{W}$ e read with interest the systematic review conducted by Piccione et al. into the clinical and functional recovery of patients with pelvic fracture (PFs). They summarized their finding into practice advice and recommendations for the rehabilitation of such patients. ${ }^{1}$ Eighty articles were included ranging from reports into the epidemiology, current treatment and rehabilitation strategy, associated traumatic injuries and complications of PFs. This review attests to the significance and indispensability of early multidisciplinary intervention in patients with PFs. However, in our view, it also left many questions unanswered, which may cause potential bias. There is uncertainty in the methodological process in terms of study selection for the review. The authors only searched three databases, ignoring the rehabilitationoriented databases and grey literature. It is, therefore, possible that eligible reports could have been omitted, leading to potential bias. The search strategy and history, which are of great importance in such a review, were missing. The study selection process, therefore, could not be replicated, decreasing the level of evidence. Notably, the study inclusion and grading criteria remained unclear, revealing no consistent standards. It seems that the authors had no prior hypothesis of the type of review they were conducting before the inclusion of publications. As for scoring criteria, the lack of a detailed description of the specific standards and the exact exclusion criteria could lead to significant methodological problems. In summary, the methodological deficiencies cannot be underestimated. In summary, given all these confounding factors, the level of evidence presented is much decreased. Consequently, potentially recommendations based on such a review could lead to inappropriate rehabilitative management and eventually worsen the long-term outcome of patients with PFs. Therefore it is unwise to give specific recommendations on the rehabilitation management of patients with PFs based on such a review. Furthermore, it is unclear how the various definition throughtout used was determined as it is not mentioned in the report. The daily clinical practice could be easily misguided due to misunderstandings without precise definitions. Last but not least, some of the proposals themselves were still in dispute. For instance, early reports have suggested opposite rehabilitation strategies over whether the weight-bearing process should start sooner, while insufficient evidence is given to update them. ${ }^{2-5}$ As protocols vary significantly from one study to another, the level of evidence and study quality should be evaluated individually to identify a more standardized guide for PFs rehabilitation. To date, the lack of high-quality meta-analysis of rehabilitation after PFs regarding types of therapy, care pathways, and 


\section{Rehabilitation of pelvic fractures needs to be further optimized}

Eur J Transl Myol 31 (4): 10246, 2021 doi: 10.4081/ejtm.2021.10246

functionality outcomes stills needs to be solved. Further studies and sustained effort are warranted to understand and optimize the multidisciplinary rehabilitation of patients with PFs.

\section{List of acronyms \\ PFs - Pelvic fractures}

\section{Authors contributions}

Zehua Li and Dilihumaer Maimaitiming contributed equally to this work. W.X and Z.L were involved in the report conception and obtaining of funding; W.X, Z.L, D.M, and L.S were involved in drafting the manuscript; all authors were involved in the critical revision of the manuscript for important intellectual content. All authors read and approved the final manuscript.

\section{Acknowledgments}

None.

\section{Funding}

This report was supported by Sichuan Science and Technology Program, No.2021YFH0141.

\section{Conflict of Interest}

The authors declare no competing interests.

\section{Ethical Publication Statement}

We confirm that we have read the Journal's position on issues involved in ethical publication and affirm that this report is consistent with those guidelines.

\section{Corresponding Author}

Weixi Xiong, Department of Neurology, West China Hospital, Sichuan University, Chengdu, 610041, Sichuan, China.

ORCID:0000-0001-6835-8664

E-mail: 502216168@qq.com
E-mails and ORCID iD of co-authors

ZehuaLi: peter.lizh@foxmail.com

ORCID iD: 0000-0002-9032-3449

Dilihumaer Maimaitiming: 1038382573@qq.com

ORCID iD: 0000-0002-8152-3254

Leqi Sun: skgtuna@ucl.ac.uk

ORCID iD: 0000-0002-8476-7588

Haoran Wang: 13540886708@163.com

ORCID iD: 0000-0001-9690-0051

\section{References}

1. Piccione F, Maccarone MC, Cortese AM, Rocca G, Sansubrino U, Piran G, Masiero S. Rehabilitative management of pelvic fractures: a literature-based update. Eur J Transl Myol. 2021 Sep 17;31(3):9933. doi: 10.4081/ejtm.2021.9933.

2. Rickman M, Link BC, Solomon LB. Patient Weight-bearing after Pelvic Fracture Surgery-A Systematic Review of the Literature: What is the Modern Evidence Base? Strategies Trauma Limb Reconstr. 2019 Jan-Apr;14(1):45-52. doi: 10.5005/ jp-journals-10080-1414.

3. Haller JM, Potter MQ, Kubiak EN. Weight bearing after a periarticular fracture: what is the evidence? Orthop Clin North Am. 2013 Oct;44(4):509-19. doi: 10.1016/j.ocl.2013.06.005. Epub 2013 Aug 1.

4. Cornwall R, Gilbert MS, Koval KJ, Strauss E, Siu AL. Functional outcomes and mortality vary among different types of hip fractures: a function of patient characteristics. Clin Orthop Relat Res. 2004 Aug;(425):64-71. doi: 10.1097/01.blo.000013 2406.37763.b3.

5. Meys G, Kalmet PHS, Sanduleanu S, van Horn YY, Maas GJ, Poeze M, Brink PRG, Seelen HAM. A protocol for permissive weight-bearing during allied health therapy in surgically treated fractures of the pelvis and lower extremities. J Rehabil Med. 2019 Apr 1;51(4):290-297. doi: 10.2340/165019772532.

Submission: October 22, 2021

Revision submitted: November 18, 2021 Accepted for publication: November 18, 2021 